\title{
Acciones de respuesta ante la Covid-19 en Brasil y Colombia: cayuda para quién?
}

\section{Brazil and Colombia's economic responses to COVID-19}

\author{
Karen V Marriner ${ }^{1}$ \\ Laura $S$ Becerra ${ }^{2}$ \\ Fecha de recepción: 27/10/2020 - Fecha de aceptación: 01/12/2020 \\ DOI: https://doi.org/10.22490/26655489.4240
}

\section{Resumen}

Este trabajo de investigación se centra en el análisis de las medidas económicas implementadas ante la actual crisis ocasionada por la pandemia por la enfermedad de la COVID-19. Concretamente en los casos de Colombia y Brasil, los países latinoamericanos que más medidas económicas implementaron en el primer semestre del 2020. En este artículo se explorarán las diferencias, semejanzas y los resultados que han tenido estas medidas, con el objetivo de analizar y discutir la orientación de las medidas económicas y de identificar ¿Qué actores/sectores se han visto beneficiados en mayor medida con la implementación de las acciones económicas? Si bien la posición de los Gobiernos de Brasil y Colombia difieren frente a la forma de afrontar la pandemia por la enfermedad de la COVID-19, en ambos casos se ha identificado que las ayudas o bien han estado dirigidas a los grandes gremios o sistemáticamente se han orientado al beneficio de un grupo minoritario, priorizando al empresariado tradicional y evidenciando que aún en esta coyuntura, las prácticas de corrupción y el aumento de la desigualdad siguen manifestándose.

$1 \mathrm{Ma}$. en Estudios para el Desarrollo del International Institute of Social Studies, de la Universidad de Erasmus Rotterdam, La Haya, Países Bajos, 2018. Docente investigadora de la Universidad Externado de Colombia. Karen.marriner@uexternado.edu.co Orcid 00000001-6612-8232

2 Estudiante de Gobierno y Relaciones Internacionales de VIII semestre de la Universidad Externado de Colombia. laura.becerra@uexternado.edu.co Orcid 0000-00025784-3178 
Palabras clave: respuesta económica, Colombia, Brasil, COVID-19, reactivación, élites.

\section{Abstract}

This article analyses the economic measures that have been implemented as a response to the COVID-19 crisis. Latin America and the Caribbean have been hit particularly hard, with an expected contraction of 9.3 percent this year-its largest recession on record. The policy response in the region has been different in every country, however, the monetary and fiscal measures are a priority. In this scenario, this document explores the measures implemented by Colombia and Brazil, in order to identify Which actors/sector have been the most benefited?

Keywords: Economic responses, Colombia, Brasil, COVID-19, reactivation, elites.

\section{Introducción}

Al igual que el resto del mundo, la economía latinoamericana enfrenta uno de los choques más fuertes debido a la actual pandemia de la enfermedad por coronavirus (COVID-19). El crecimiento para este año en la región, según la Comisión Económica para América Latina y el Caribe (CEPAL), se proyectaba en una senda de bajo crecimiento, con una expansión estimada del producto interno bruto (PIB) de 1,4\% (CEPAL, 2019). Sin embargo, finalizando el primer semestre del año el panorama supera cualquiera de las expectativas pesimistas. La misma entidad proyecta una contracción de la actividad económica hasta del 5,3\%, la peor que la región ha sufrido desde el siglo anterior. En cuanto al PIB per cápita se señala una década perdida pues se estima volver a los niveles alcanzados en el 2010. 
Figura 1. Evolución PIB per cápita en América Latina

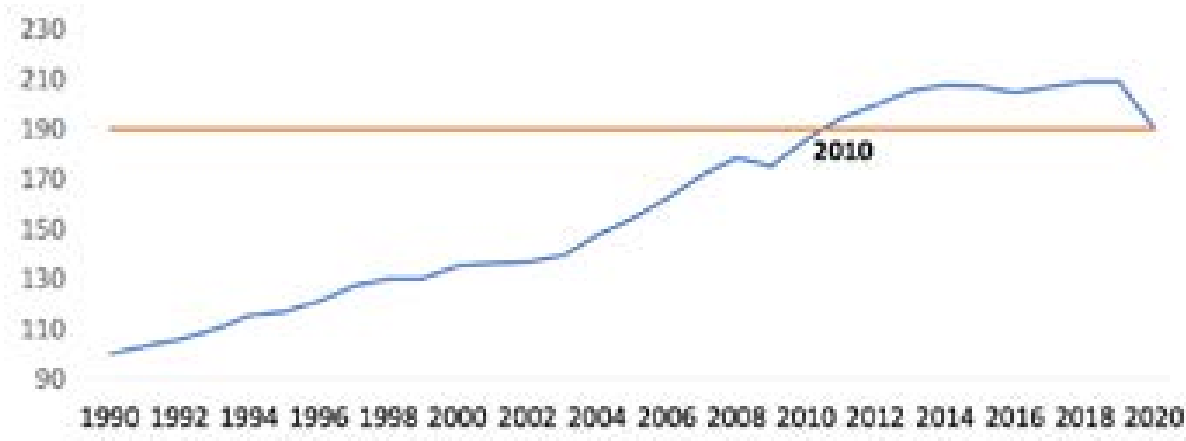

Fuente: CEPAL, 2020a

Bajo ese contexto, los Gobiernos nacionales han debido cambiar de prioridades para hacer frente a la coyuntura actual: "La pandemia ha obligado a todos los actores a redefinir sus planes y reencausar los recursos disponibles para poner en el centro de su accionar no solo la Agenda 2030 para el Desarrollo Sostenible, es decir avanzar hacia la sostenibilidad económica, social y ambiental sin dejar a nadie atrás, sino también las respuestas a la crisis sanitaria, económica y social" (CEPAL, 2020a, p. 13)

Algunas de las estrategias implementadas se han focalizado en establecer medidas de apoyo económico para reactivar la economía y evitar profundizar aún más la crisis. En ese sentido, el objetivo de este trabajo es analizar y discutir la orientación de las medidas económicas implementadas en el marco de la actual crisis, con el ánimo de identificar qué actores se han visto beneficiados. Para esto, el análisis se centrará en los países suramericanos que han impulsado más acciones de apoyo económico, a saber: Brasil y Colombia.

El documento se divide en 5 partes incluyendo la presente introducción. En primer lugar, se presentará el contexto y los impactos que la pandemia por la COVID-19 han causado en la región latinoamericana. La sección 2 describe las nuevas incertidumbres que ha traído la pandemia. Luego, las secciones 3 y 4 analizarán las medidas económicas implementadas en Colombia y en Brasil, respectivamente, con el ánimo de identificar cuáles han sido los beneficios. Finalmente, la sección 5 presentará las consideraciones finales. 


\section{Covid-19 y nuevas incertidumbres}

En un escenario de incertidumbre como el que trajo la pandemia por la enfermedad de la COVID-19 se tiene como certeza que ningún país de América Latina estaba preparado para afrontar los efectos de una crisis sanitaria con repercusiones en el ámbito económico, social y político, de la envergadura como las que se han presentado. La COVID-19 ha profundizado las ya conocidas deficiencias de los países latinoamericanos La desigualdad continua y creciente ya estaba presente en casi todos los países y se ha visto como los vulnerables son los que han afrontado mayores consecuencias: las personas que dependen de la economía informal, las mujeres, las personas con discapacidad, los refugiados y desplazados, y los que padecen estigmatización. El virus ha mostrado la vulnerabilidad de ciertos grupos pues los riesgos por mayor exposición varían de acuerdo con las condiciones económicas, principalmente. Según el PNUD (PNUD, 2020), el mayor riesgo lo asumen las personas que actualmente viven en la pobreza. A pesar de los recientes avances en su reducción, alrededor de una de cada cuatro personas todavía vive en situación de pobreza multidimensional o es vulnerable a ella, y más del 40 por ciento de la población mundial carece de protección social alguna. La tabla 1 muestra las proyecciones de pobreza para los países latinoamericanos. Se resalta que la pobreza aumentará de 186 a 231 millones de personas, y 96 millones de personas estarán en condiciones de pobreza extrema. Los incrementos más representativos se presentarán en Argentina, Perú, Brasil y El Salvador. 
Tabla 1. América Latina: proyección de la población en situación de pobreza y pobreza extrema en 2020

\begin{tabular}{|c|c|c|c|c|c|c|}
\hline & \multicolumn{3}{|c|}{ Pobeeza Extrema } & \multicolumn{3}{|c|}{ Pobseas } \\
\hline & $2515 \mathrm{~b} / \mathrm{f}$ & $2020 \mathrm{~d}$ & merements as & $2019 \mathrm{~b} /$ & 2000 of & minemen \\
\hline Argentina af & 1.8 & 69 & 21 & 267 & 375 & 103 \\
\hline 9olvis & 14.3 & 16.8 & 25 & 323 & 36.1 & 3.8 \\
\hline Brasil & s.s & 9.8 & 43 & 19.2 & 269 & 7,7 \\
\hline Chile & 1.4 & 3.4 & 2.0 & 9.8 & 15.5 & 5.7 \\
\hline Colombla & 10.3 & 14.3 & 4.0 & 200 & 34: & 5.2 \\
\hline Costa Rea & 1.4 & 5.1 & 17 & 165 & 205 & 40 \\
\hline Cowedor & 7.6 & 127 & 5.1 & 25.7 & 32.7 & 70 \\
\hline El salvader & 7.4 & 119 & 45 & 33.7 & 402 & 6.5 \\
\hline Gusetmala & 19.8 & 22.7 & 29 & 48.6 & $\$ 1.6$ & 30 \\
\hline Mendurat & 15.7 & 22.2 & 15 & $54:$ & 500 & 4.2 \\
\hline Metrico & 11.1 & 174 & 63 & 419 & 495 & 76 \\
\hline Nicarapua & 180 & 228 & 48 & 471 & 52.7 & 5.6 \\
\hline Panami & 65 & 85 & 20 & 14.6 & 275 & 29 \\
\hline Rarisulyr & 6.2 & 6.6 & 0.4 & 19.4 & 209 & 1.5 \\
\hline Aend & 27 & 7.6 & 29 & 16.5 & 28.8 & 9.3 \\
\hline Arpublica Dominicans & es & 6.7 & 22 & 203 & 24.7 & 4,4 \\
\hline Unuguary & a1 & as & 02 & 2.9 & 5.3 & 2.4 \\
\hline
\end{tabular}

Fuente: Cepal, 2020a

En ese contexto, los Gobiernos han tenido que definir varias estrategias para enfrentar la crisis. Estas medidas van desde la imposición de cuarentenas y restricciones a la movilidad, hasta el cierre de fronteras y aeropuertos. Muchos han señalado la pandemia como una oportunidad en la que "los gobernantes ya proclives al autoritarismo tienen la oportunidad de reducir libertades y afianzarse bajo la máscara de la urgencia" (Revista Semana, 2020), e incluso implementar políticas que en otros contextos hubieran presentado gran resistencia en su implementación. Esta situación se ha relacionado con la "doctrina del shock" y el "capitalismo catástrofe", términos que se refieren a cómo ciertos grupos emergen y se benefician de las crisis y las medidas adoptadas en esas coyunturas. "La doctrina del shock es la estrategia política que consiste en emplear las crisis a gran escala para hacer avanzar políticas que profundicen sistemáticamente las desigualdades, enriqueciendo a las élites y debilitando a los demás"(Klein, 2008, p. 26).

La pandemia causada por la COVID-19 se ha presentado como una oportunidad de poner en práctica la doctrina del shock. Más aún cuando, dada la emergencia que presenta la coyuntura, los gobiernos pueden "acelerar la aprobación de las leyes coordinando con el presidente, quien las presenta con "carácter 
de urgencia" al Congreso, además de implementar las leyes de acuerdo con un cierto criterio que favorece a las élites económicas al priorizar las grandes inversiones"(Durand, 2016, p. 28).

Los temas medio ambientales constituyen uno de los grandes ejemplos de los retos y dilemas que han profundizado la crisis por la pandemia. Para ilustrar, "las industrias más contaminantes alrededor del mundo están usando la pandemia para ganar billones de dólares a través de las "ayudas" que han terminado por debilitar y retrasar la protección a los recursos del medio ambiente" (Carrington, 2020). Así pues, se han presentado varios cuestionamientos sobre qué debe primar: ¿̇los años de esfuerzos por consolidar la protección ambiental y aprovechar esta oportunidad para avanzar hacia la agenda 2030, o privilegiar y apoyar sectores tradicionalmente contaminantes para evitar una mayor crisis económica? De igual forma, las medidas implementadas por diferentes países llevan implícitas decisiones en donde se deben priorizar ciertos intereses sobre otros; por ejemplo: ¿recuperar la economía con la reactivación de la vida "normal" o continuar con políticas restrictivas para evitar el contagio?, ¿apoyar a los grandes empresarios o a los pequeños productores? O simplemente no intervenir.

Dentro de América Latina se han identificado diferentes formas de enfrentar y asumir pandemia. Por ejemplo, mientras que la mayoría de los países hace pública la información sobre los contagios y casos, hay países que han decidido limitar el acceso a la información. Algunos de los casos más notorios de ello son El Salvador y México, cuyos gobiernos tomaron la decisión de suspender los procedimientos para solicitud de información hecha en el marco de leyes de acceso de información pública, citando la urgencia de la respuesta a la pandemia. Además, es relevante señalar las diferentes condiciones en las que se ha enfrentado la crisis: "muchos ejecutivos poseen un escaso liderazgo social (Chile), afrontan una nueva crisis económica de enorme magnitud (Argentina), se encuentran en el tramo final de sus mandatos (Perú y Ecuador), se enfrentan a una campaña electoral muy polarizada (Bolivia) o cuentan con unas administraciones públicas muy débiles (la mayoría de Centroamérica y el Caribe) o están sumidos en un fuerte deterioro institucional y económico (Nicaragua y Venezuela) o son Estados fallidos (Haití)" (Real Instituto Elcano, 2020). 


\section{Medidas económicas implementadas en Colombia y Brasil}

Los impactos económicos no se hicieron esperar y los gobiernos se han visto obligados a establecer medidas que impidieran profundizar aún más las consecuencias de la crisis. La figura 2 muestra el número de medidas implementadas por país, en donde se destaca que Brasil y Colombia han reaccionado de forma activa priorizando la intervención a través de acciones económicas, mientras que países como Ecuador, Venezuela, Guyana y Surinam, apenas han implementado algunas medidas.

\section{Figura 2. Número total de acciones económicas} implementadas por país 2020-I ${ }^{3}$

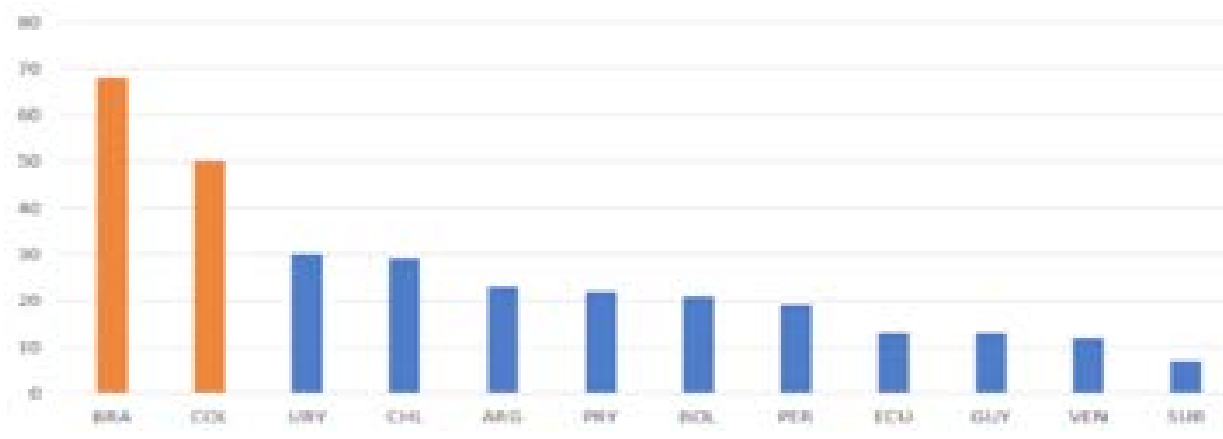

Fuente: Elaboración propia a partir de datos de CEPAL, 2020b

Asimismo, de acuerdo con el Government Response Tracker de la Universidad de Oxford, estos dos países presentan un índice de medidas económicas ${ }^{4}$ que supera el promedio de los países latinoamericanos. Se puede ver que, en el caso de Colombia, desde abril este país ha presentado el mayor índice de apoyo de la región. Por su parte, Brasil comenzó a subir en el índice solo a partir de junio, pero alcanzando el nivel de Colombia rápidamente. En este punto es relevante señalar que, aunque ambos países presentan niveles similares en cuanto al número de medidas, la posición de cada uno ha sido diferente, pues mientras que el presidente Bolsonaro es reconocido como uno

3 La información presentada corresponde al primer semestre de 2020. Fecha de corte: 30 de junio de 2020

4 El índice de economic measures es un indicador que mide: el apoyo al ingreso, medidas de alivio en deuda para los hogares, medidas fiscales y apoyo a otros países. 
de los líderes más escépticos sobre la gravedad de la pandemia, el presidente colombiano ha tenido una actitud proactiva frente a la COVID-19.

Figura 3. Economic Index, Brasil y Colombia

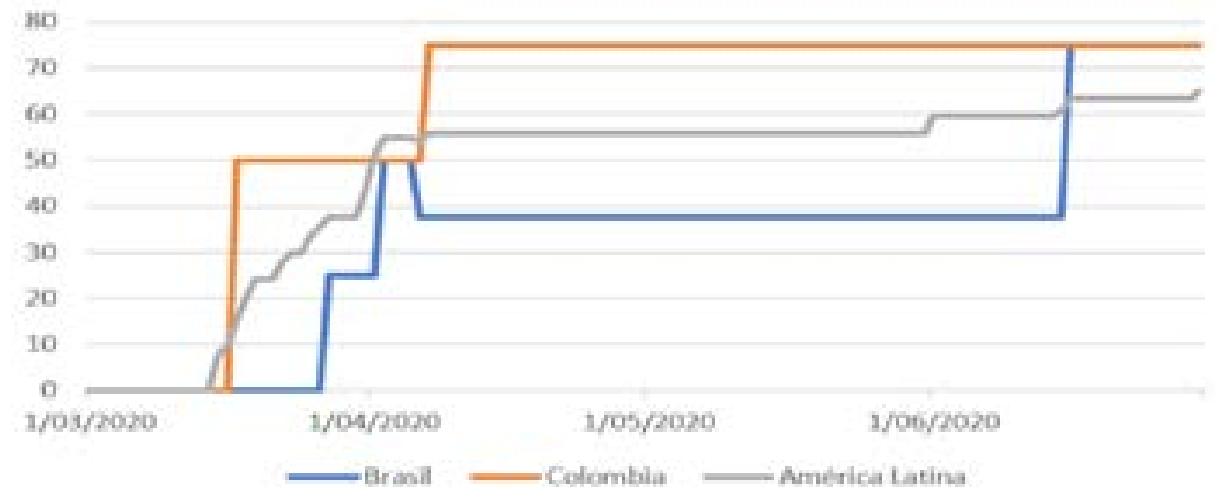

Fuente: Elaboración propia con datos de la University of Oxford, 2020

Sin embargo, aún con las medidas implementadas, las predicciones económicas no mejoran. En el caso colombiano, el Dane informó que el desempleo de Colombia en julio de 2020 fue de $20,2 \%$, cifra superior en casi 10 puntos porcentuales al $10,7 \%$ registrado en el mismo mes del 2019. Asimismo, las condiciones en Brasil son preocupantes, la tasa de desempleo subió a $13,8 \%$ en el trimestre mayo-julio, un récord desde el inicio de la serie histórica en 2012.

Bajo ese contexto, la respuesta de los países de la región se ha enfocado en medidas como el suministro de liquidez, la flexibilización de los requisitos de reserva para los bancos, reducciones en las tasas de interés, el establecimiento de líneas de crédito, intervención en el mercado cambiario, entre otros. Muchos países han anunciado paquetes de estímulo fiscal que incluyen asistencia social, apoyo para pequeñas empresas, fondos adicionales para el sector de la salud, la postergación de los vencimientos de impuestos y la suspensión de pagos de préstamos y servicios públicos. En esta sección se revisarán las acciones económicas que tanto Brasil como Colombia han implementado. 


\subsection{Colombia}

Según las perspectivas de crecimiento para el 2020, Colombia se iba a mantener como uno de los países con mayor crecimiento de la región. Sin embargo, finalizado el primer semestre del año, las condiciones socioeconómicas del país son preocupantes. En los escenarios más pesimistas se estima una caída de la economía hasta del $-7,9 \%$. Además, el desempleo sobrepasó el $20 \%$ y se ha enunciado que podría aumentar al 21,5\% (Mejía, 2020). Esto significa que podría haber dos millones y medio de nuevos desempleados. Asimismo, la Superintendencia de Sociedades presentó un balance de las solicitudes de insolvencia que se han radicado durante la emergencia por el coronavirus y que se espera que en los próximos meses se presenten picos de solicitudes como consecuencia de la crisis.

Así mismo, teniendo en cuenta que un "47 \% de los trabajadores está en la informalidad, que tan solo dos de cada diez hogares cuentan con ahorros para cubrir un imprevisto, y que el sistema laboral está especialmente precarizado, lo que afecta incluso a profesionales con un alto nivel de formación y al personal sanitario", las acciones económicas que se implementen son de vital importancia para mantener a flote no solo la economía del país sino las vidas de los colombianos.

El primer caso de Colombia se registró el 6 de marzo de 2020 y una semana después comenzaron a tomarse acciones por parte del Gobierno nacional. Según el Observatorio COVID-19 de la CEPAL, Colombia ha implementado 50 acciones de alivio económico ${ }^{5}$ (corte a 30 de junio de 2020). La figura 4 presenta el número de medidas clasificadas de acuerdo con el propósito. Como se puede observar, en el caso colombiano la mayoría de las medidas son del tipo política empresarial, política monetaria y política fiscal.

5 En total se han implementado 112 acciones en temas como educación, género, protección social, medidas laborales, etc. Empero, como se denota, casi la mitad de las acciones han sido de corte económico. 


\section{Figura 4. Medidas económicas implementadas por Colombia 2020-I}

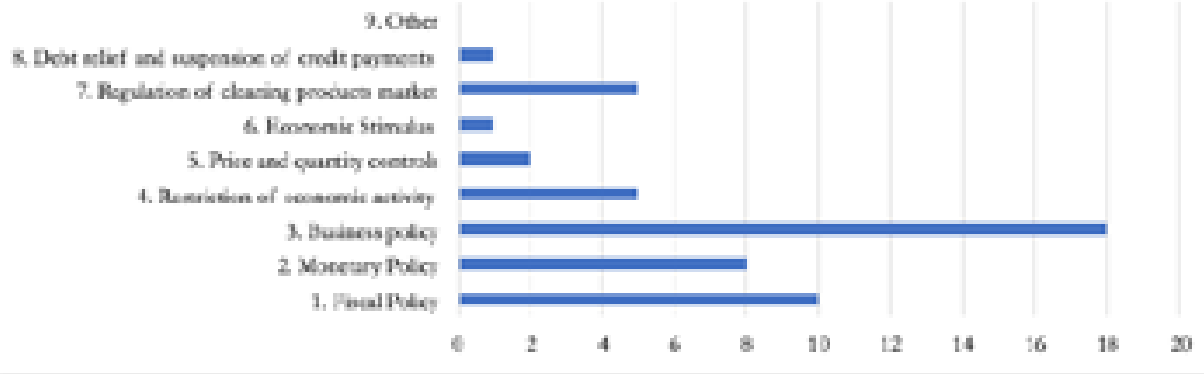

Fuente: Elaboración propia con datos de la CEPAL, 2020b

En términos de política fiscal, tanto el Gobierno nacional como los gobiernos locales expandieron los plazos para pagar las obligaciones tributarias. Asimismo, se cambió el calendario para la declaración y pago de impuesto sobre la renta y complementarios para grandes contribuyentes, la sobretasa de las entidades financieras, la declaración de activos en el exterior, el impuesto al consumo y el impuesto sobre las ventas (IVA). Frente a este último, el Gobierno anunció que se realizarían 3 días sin IVA: "Es el nacimiento oficial de esos tres días sin IVA, que están siendo pensados para el 19 de junio, 3 julio y 19 de julio y, por supuesto, se busca que tengamos la adquisición de esos bienes, ya sea por vía electrónica o vía presencial" (Presidencia de la República de Colombia, 2020).

Al analizar los días sin IVA con mayor detalle, y de paso rectificar sí cumplieron su objetivo de recuperar la demanda y la economía del país (Presidencia de la República de Colombia, 2020c), se puede decir que fueron más los costos asumidos por el Gobierno y los ciudadanos que las ganancias arrojadas por estas medidas. En un primer plano, la reactivación pareció haber sido un éxito, pues las ventas del comercio mostraron un crecimiento del $158 \%$ y se superaron los $\$ 5$ billones en ventas, de los cuales los bienes de entretenimiento y electrodomésticos, equipos y aparatos de sonido y video, artículos deportivos y productos textiles y calzado, fueron los más adquiridos por los colombianos (Presidencia de la República de Colombia, 2020b). Además de los beneficios de las ganancias que recibieron los comerciantes por las ventas, se esperaba que el sector manufacturero también tuviera beneficios con la medida pues las compras ayudarían a 
mover los inventarios de las empresas comerciales y, de esta manera, las fábricas deberían producir más bienes (Galán, 2020). No obstante, el efecto no fue el esperado. Para el mes de agosto, el Indicador de Seguimiento a la Economía (ISE) 6 cayó $-1,19 \%$ respecto a julio, en el que el sector secundario presentó una reducción del $-12,2 \%$, principalmente por la desaceleración de la manufactura (DANE, 2020b; Portafolio, 2020a). Por otra parte, el súbito aumento de las ventas de electrodomésticos y bienes tecnológicos pueden explicar el aumento del déficit de la balanza comercial en un $52 \%$, llegando a ser de $-901,5$ millones de dólares en julio y $-827,9$ millones de dólares en agosto (DANE, 2020a). Con este panorama es claro que si bien los comerciantes, especialmente de las grandes superficies, lograron reactivar su actividad económica, el beneficio no impactó la industria colombiana sino la industria extranjera a costa del gasto público de aproximadamente $\$ 400000$ millones de pesos (Morales, 2020), lo que terminó estimulando muy poco a la producción doméstica.

Frente a las acciones en materia de política monetaria, se han bajado las tasas de interés en tres ocasiones. En total, la disminución ha sido de 4,25 \% a 2,75 \%. De hecho, ha sido tal el esfuerzo del Banco de la República (BanRep) por reactivar la economía, que en septiembre se bajó la tasa a 1,75\%, el nivel más bajo en la historia de Colombia (La República, 2020). Efectivamente, las tasas de interés se redujeron respecto al 2019, pero esta reducción fue más amplia para las empresas que para las personas. De acuerdo con datos de la Superintendencia Financiera, la variación de las tasas entre el 6 de marzo y el 9 de octubre del 2020, para las empresas fue del $-353 \%$, mientras que para las personas fue de $-195 \%$ en el caso de la modalidad de crédito a través de tarjeta, y en el caso de las microempresas, las tasas para microcréditos tuvieron una variación del 212 \% (Superintendencia Financiera de Colombia (SFC), 2020). Sistemáticamente, era de esperar que el primer beneficiario de la acción implementada por el Banco Central fueran las empresas, pues estas manejan los créditos de muy corto plazo y que por el riesgo que supone desmejorar la calidad de la cartera de las entidades financieras, estas subieran los intereses en lugar de bajarlos (Portafolio, 2020b). Con estas facilidades para las empresas, la eficiencia de la asignación de

6 El ISE calcula el comportamiento de múltiples actividades económicas, tales como minería, actividades agropecuarias, industriales, de servicios, entre otras. 
recursos por la que propende el sistema financiero empieza a verse perjudicada al igual que la transmisión de la política monetaria, teniendo en cuenta que "el acceso y la utilización efectiva de los productos financieros son un vehículo eficiente para suavizar el ingreso y los ciclos del consumo" tanto de los hogares como de las empresas (Cano et al., 2014).

Asimismo, otra de las medidas de política monetaria que destacan tiene que ver con la suspensión de compra de bonos del tesoro y a la compra de 8 billones de pesos en TES y 2 mil millones de dólares para la reserva. Se realizaron operaciones de venta de divisas a futuro y de swaps, con el fin de dar liquidez a la economía. También se creó el Fondo de Mitigación de Emergencia (FOME), la transferencia de los dividendos de las empresas públicas al Fondo Nacional de Garantías y el decreto de un aporte por parte de los empleados públicos y contratistas que ganan entre 10 y 15 millones de pesos (más o menos 2 360 euros) aportarán el $10 \%$ y los que devengan más de 15 millones de pesos contribuirán con el $15 \%$. En general, estos recursos públicos canalizados tienen como objetivo "atender las necesidades de recursos para la atención en salud, los efectos adversos generados por la actividad productiva y la necesidad de que la economía continúe brindando condiciones que mantengan el empleo y el crecimiento" (Decreto 444, 2020), lo que se propone realizar a través del pago de costos generados por contratos en pro del objetivo, la realización de operaciones de apoyo de liquidez al sector financiero, proveer financiamiento a empresas privadas, públicas o mixtas que desarrollan actividades de interés nacional, entre otras. Para lograr lo propuesto, el Gobierno nacional logró sumar un total de $\$ 25,5$ billones de pesos (Observatorio Fiscal de la Pontificia Universidad Javeriana, 2020).

Si bien la inversión de recursos ha sido notable y han ayudado a disminuir los efectos socioeconómicos de la pandemia, la administración de estos recursos no ha sido transparente del todo. Para comenzar, entre el periodo de marzo a julio se recibieron 72 reportes de irregularidades en el manejo de los recursos en el Centro de Asesoría Legal Anticorrupción de la ONG Transparencia Internacional en Colombia, de los que más de la mitad corresponden a irregularidades en la contratación, 7 casos corresponden al mal manejo y desvío de recursos en la entrega de productos contratados, como mercados, y 7 casos 
están relacionados con el uso indebido de apoyos económicos (Transparencia Internacional, 2020b, 2020a). También se recibieron reportes por irregularidades por sobrecostos, favorecimiento de los intereses políticos, entre otros. Una de las tantas irregularidades identificadas fue la adjudicación de contratos a 331 personas jurídicas y naturales que financiaron campañas políticas en 2019, contratos cuyos valores superan, aproximadamente, 8 veces los aportes hechos en campaña.

Asimismo, el reporte realizado por la Alianza "Ciudadanía Activa con los ojos puestos en los recursos de la COVID-19" revela que, al analizar los contratos realizados por contratación directa bajo la causal de urgencia manifiesta, algunos contratistas no cumplían con las condiciones básicas para ser idóneos, como no contar con experiencia o presentar incongruencias entre el objeto social de la empresa y el objeto del contrato. (Transparencia Internacional, 2020a). Paralelamente, la falta de claridad sobre el gasto también se deriva tanto de la carencia de un sistema transparente y público que exponga de manera detallada y clara la inversión de los recursos como de un plan de gastos detallado, exigido por la Ley de transparencia (Ley 1712 de 2014), en los que se desglose la fuente y la destinación de los recursos (Observatorio Fiscal de la Pontificia Universidad Javeriana, 2020). Esta pandemia ha demostrado que por más que el Congreso limite la función pública para evitar los actos de corrupción, esto no es suficiente para romper con las estructuras Estado-Empresarios que están presentes en los territorios; de acuerdo con un estudio realizado por Gallego, Prem y Vargas (Gallego, Prem and Vargas, 2020), en el cual se analizaron casi 360000 contratos realizados en el primer semestre del año a causa de la emergencia sanitaria, pudieron evidenciar que en los municipios con menor capacidad institucional y menor transparencia, se incrementaron el número y el valor de los contratos con irregularidades procedimentales.

Bajo ese marco, se pueden identificar las estrechas relaciones entre los administradores del gasto y los empresarios que han financiado sus puestos; una vez más, el capitalismo clientelista se aprovecha de las carencias sociales y de la desigualdad para comenzar a definir el destino político del país bajos los intereses de unos grupos específicos. Aunque esta premisa no es una novedad, sí vale la pena subrayar que la corrupción en este contexto ha 
resultado ser un mal más grave que el coronavirus (Ávila, 2020), pues la recuperación económica y social se está viendo rezagada y distorsionada por el saqueo de los recursos públicos.

En cuanto a las medidas de política empresarial, resaltan la creación de nuevas líneas de crédito, generales y específicas para el sector turismo y agricultura. Además, se estableció una inversión en el sector de aviación por 71214 millones de pesos. Ampliación de créditos condonables ligados al compromiso de no realizar despedidos de nómina; reestructuración de deudas y establecimiento de tarifas diferenciales para las pequeñas y medianas empresas para que puedan obtener préstamos y otras ayudas financieras. En este punto es necesario destacar que, en lo corrido del periodo de aislamiento, se han efectuado 143 555567 operaciones de créditos desembolsados, por un valor total de $\$ 174,95$ billones, monto del cual, $\$ 111,81$ billones de pesos se han desembolsado a las empresas, lo que contrasta con el monto desembolsado a las microempresas que fue de $\$ 2.28$ billones (Superintendencia Financiera de Colombia (SFC), 2020), siendo una cifra 20 veces menor a lo que fue concedido en créditos para personas naturales en tarjetas de crédito y otros consumos. Como se mencionó anteriormente, una de las grandes preocupaciones de los bancos al conceder préstamos era el alto riesgo que este implica, por lo que el Gobierno nacional decidió capitalizar el Fondo Nacional de Garantías, el cual tiene como objetivo facilitar la obtención de créditos por parte de pequeñas y medianas empresas (pymes) por medio de la garantía de que si el prestatario no logra pagar su crédito, el Gobierno paga entre el 50 $\%$ y el $90 \%$ del crédito (Presidencia de la República de Colombia, 2020a). Como resultados, la Superintendencia Financiera reportó que, al 11 de octubre, se habían negado 48714 solicitudes por un valor de $\$ 5,6$ billones, de las cuales 48669 solicitudes hechas por pymes, microempresas y trabajadores independientes fueron rechazadas, lo que configuró un promedio de tasa de aprobación de créditos del $77.32 \%$ para las pymes, lo que contrasta con el promedio de tasa de aprobación para grandes empresas que fue del 87,23 \%. Efectivamente, como lo mencionó el presidente Duque, para la microempresa queda mucho por hacer desde las entidades financieras, quienes ya señalaron que deberán aumentar tasas de interés o reducir los préstamos a las empresas por la aversión al riesgo (Presidencia de la República de Colombia, 
2020d). No obstante, esta estrategia del Gobierno deja entrever que ni siquiera el riesgo para una entidad bancaria disminuye en un préstamo, aunque el Gobierno nacional dé garantías.

La reapertura de la economía después de la cuarentena total y obligatoria impuesta por el Gobierno nacional se ha venido dando por sectores. Los primeros sectores en retomar labores fueron el sector de la construcción y la industria manufacturera. Una de las medidas más destacadas fue la decisión de asumir las nóminas de las pymes por tres meses. Además, debe rescatarse el Programa de Ingreso Solidario Único (PIS), el cual da una ayuda de 160000 pesos (40 euros) a 3 millones de familias identificadas a partir de los datos del SISBEN. Los programas de ayuda a las familias más necesitadas cubren a unos 6 millones de familias, pero los datos indican que las familias vulnerables podrían llegar a los 10 millones. Aunque fue un programa diseñado y puesto en marcha en tiempo récord, las fallas del PIS podrían estar causando la exclusión de estos beneficios a poblaciones tan vulnerables como madres cabezas de hogar, según relata DeJusticia (DeJusticia, 2020), ya que los criterios para escoger a los beneficiarios no son claros. En cifras, aproximadamente un millón de familias podrían dejar de percibir este subsidio del gobierno.

Mientras tanto, la mayoría de las medidas benefician a las grandes empresas que cumplen los requisitos para acceder a las líneas de crédito. Esto fue evidente con la línea de crédito destinada para el sector agropecuario, la cual tenía como objetivo ayudar al mediano y pequeño empresario y al campesino de a pie, pero según la Contraloría de esos $\$ 226$ mil millones en créditos, el $94 \%$ fue para grandes empresarios, $4 \%$ para medianos y tan solo $2 \%$ para los verdaderos campesinos:

"(Los recursos) se colocan sin controlar la afectación por la emergencia y su destinación, de tal manera que no surte efecto lo dispuesto por la CNCA, que estableció que los grandes clientes no pueden acceder a la línea para comercialización (...) A la fecha la línea no les está llegando a los productores agropecuarios, sino a grandes agroindustriales, comercializadores y prestadores de servicios (...) Otro elemento del desequilibrio relacionado con la destinación de estos recursos se refleja en el hecho de que Bogotá concentra 
el $35.6 \%$ de las colocaciones de emergencia, mientras que solo el $24 \%$ de municipios han tenido acceso al crédito" (Contraloría General de la República, 2020)

Además, cuando se revisa quiénes son los beneficiados de estos créditos (ver tabla 2), se evidencia que los grandes grupos empresariales y las empresas pertenecientes a las familias más poderosas económicamente son los grandes beneficiados, por ejemplo, Ingenio Providencia e Ingenio del Cauca, son empresas que pertenecen a la Organización Ardila Lulle, uno de los principales conglomerados empresariales de Colombia y de América Latina. También se destacan las empresas del grupo Avidesa pues hacen parte de las 120 empresas más grandes del país y el conglomerado Alimentos Polar.

\section{Tabla 2 Asignación línea de crédito Finagro - Principales beneficiarios}

\begin{tabular}{|l|l|r|}
\hline AVIDESA MAC POLLO & Grande productor & 10000000000 \\
\hline ORF. SA & Grande productor & 10000000000 \\
\hline AVIDESA DE OCCIDENTE & Grande productor & 10000000000 \\
\hline ITALCOL SA & Grande productor & 8100000000 \\
\hline ALIMENTOS POLAR & Grande productor & 6519878102 \\
\hline PROMOTORA DE CAFÉ COLOMBIA & Grande productor & 5000000000 \\
\hline PAPELES NACIONALES & Grande productor & 5000000000 \\
\hline AGROINDUSTRIAL MOLINO SONORA & Grande productor & 4300000000 \\
\hline FEDEARROZ & Grande productor & 4000000000 \\
\hline JOLI FOOD & Grande productor & 2875000000 \\
\hline INGENIO PROVIDENCIA & Grande productor & 2500000000 \\
\hline INVERSIONES JV & Grande productor & 2380000000 \\
\hline INGENIO DEL CAUCA & Grande productor & 2250000000 \\
\hline IMPOCOMA & Grande productor & 2250000000 \\
\hline
\end{tabular}

Fuente: Elaboración propia con datos de El Tiempo, 2020

\subsection{Brasil}

La CEPAL preveía un crecimiento por encima del 1,5\% para Brasil en el 2020. Empero, con la actual crisis sanitaria y "de acuerdo con una previsión reciente de Société Générale, la contracción de la economía brasilera será del 7,4\% y la deuda representará el 100 $\%$ del producto interno bruto en 2022" (Hernández, 2020). Si bien, 
las medidas implementadas por la Administración Bolsonaro son mucho más laxas, en términos de aislamiento pues no se ha hecho una cuarentena obligatoria, sino que se han implementado medidas de aislamiento leve, las consecuencias' a nivel económico por la pandemia por COVID-19, son preocupantes. "La combinación de inestabilidad política y catástrofe sanitaria amenaza con volverse explosiva para una economía tambaleante. Los indicadores y proyecciones sugieren que esta será la peor recesión que haya vivido Brasil" (Deutsche Welle (DW), 2020).

Bajo ese contexto, según el Observatorio COVID-19 de la CEPAL, Brasil ha implementado 68 acciones de alivio económico ${ }^{7}$, con corte a 30 de junio de 2020. La figura 5 presenta el número de medidas clasificadas de acuerdo con el propósito. Como se puede observar, al igual que en el caso colombiano, la mayoría de las medidas son del tipo política empresarial, política monetaria y política fiscal. Sin embargo, es relevante señalar que mientras Colombia comenzó a implementar las medidas desde marzo después de reportar el primer caso, Brasil desde principios de febrero y aún antes de registrar su primer caso, ya estaba definiendo acciones y medidas.

Figura 5. Medidas económicas implementadas por Brasil 2020-I

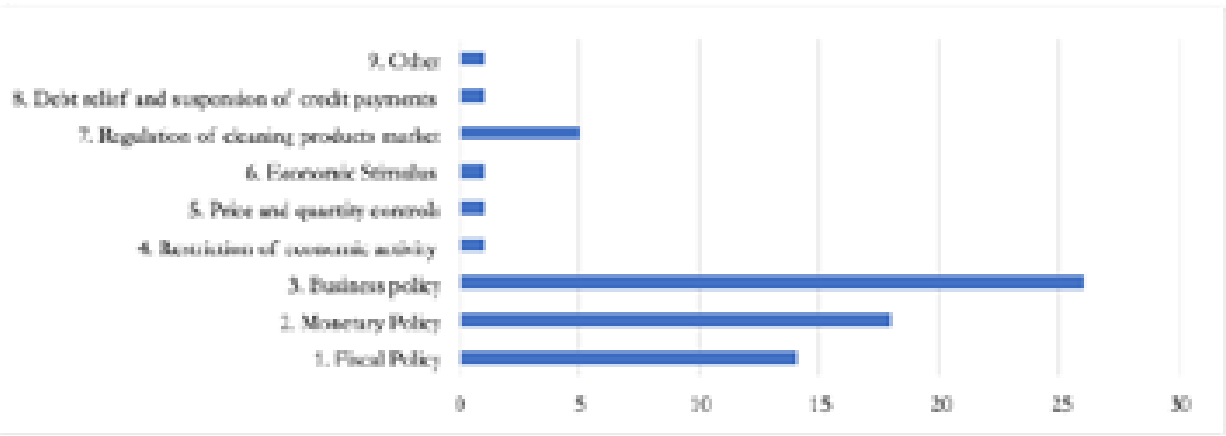

Fuente: Elaboración propia con datos de CEPAL, $2020 \mathrm{~b}$

Frente a las acciones en política fiscal se resalta la reducción de parafiscales y contribuciones de seguridad social. "El Ministerio de Economía estima que esta medida ahorrará R\$ 2,5 billones en contribuciones de las compañías" (CEPAL, 2020b).

7 En total se han implementado 250 acciones en temas como educación, género, protección social, medidas laborales, etc. 
También se eximieron varios productos de salud y de la industria manufacturera del pago del impuesto IPI. En lo que respecta a los gobiernos subnacionales, el Gobierno federal realizó transferencias intergubernamentales hacia los 17 estados equivalentes al 1,1 $\%$ del PIB de 2019 y complementó la medida con la interrupción del pago de la deuda de a los que estos son acreedores, lo que permitiría disponer de 12600 millones de reales (un 0,2 \% del PIB de 2019) para el fortalecimiento de la red de atención en salud a nivel local (CEPAL, 2020a). No obstante, estos recursos no fueron plenamente destinados a la atención de la pandemia, sino que fue la oportunidad para muchos funcionarios públicos para calibrar y nutrir sus redes clientelistas. Por un lado, los recursos para la administración de los hospitales padecieron de sobreprecios, irregularidades en la contratación, desvío de recursos, entre otros males, en la construcción y administración de hospitales de campaña en diferentes ciudades del país. Muchas de las empresas contratadas para esta titánica labor no tenían el capital suficiente para cumplir con la labor, como fue el caso de la empresa Hospital Serviços de Assistência Social Sem Alojamento LTDA, o ya tenían antecedentes de corrupción, como la empresa Iabas, la cual, en una década ha recibido por parte del municipio de Río de Janeiro un total de 812 millones de dólares en contratos por los que fueron encarcelados o multados sus administradores (Souza, 2020).

Otras de las medidas en materia fiscal que implementó el Gobierno de Bolsonaro se refieren al financiamiento de la infraestructura turística nacional, garantías para créditos y capitalización y crédito de la CEF para sectores agrícolas y se estableció un paquete de ayuda a las aerolíneas:

1. Aplazamiento de 6 meses de la recaudación de las tarifas de navegación aérea.

2. Aplazamiento hasta diciembre de 2020 de la recaudación de las tarifas de los concesionarios del aeropuerto sin multas.

3. Extensión por 12 meses para que las compañías reembolsen a los clientes los vuelos cancelados.

Además, el Gobierno Federal autorizó a las empresas a suspender por 60 días los contratos laborales y reducir las horas de trabajo por 90 días. Los trabajadores afectados serán cubiertos por el beneficio de auxilio de renta de emergencia. Las compañías podrán realizar compensaciones adicionales a los empleados, además de los auxilios gubernamentales. Las 
compañías que se adhieran a este sistema deberán mantener a los empleados de licencia, por lo menos, el mismo tiempo una vez acabe el estado de calamidad sanitaria.

Estos paquetes de medidas fiscales reflejan que, a pesar de su nivel de endeudamiento y su resultado global, Brasil aún tiene una gran capacidad de movilizar recursos a través del mercado financiero interno (CEPAL, 2020c) Sin embargo, al igual que en caso de Colombia, las facultades excepcionales concedidas a los estados para adquirir bienes y servicios de una manera más laxa, ha configurado una ventana de oportunidad para la contratación indebida y la malversación de recursos. Según cifras de la Contraloría General Federal, se han iniciado 38 investigaciones para revisar contratos fraudulentos por más de $R \$ 700$ millones de reales, lo que equivale a 124 millones de dólares (Controladoria-Geral da União, 2020).

Las acciones referentes a política monetaria se concentran en la disminución de la tasa de interés, tanto macroeconómica, la cual alcanzó un límite histórico del 3,75 \%, como de instrumentos financieros como en las tarjetas de crédito y créditos de libre inversión. Asimismo, se disminuyó el monto mínimo de reserva obligatoria para los bancos y se establecieron lineamientos para la reestructuración de deudas de empresas e individuos. En medio de la emergencia, el 7 de mayo el Congreso ratificó una enmienda constitucional para permitir al Banco Central implementar un programa de Quantitive Easing (QE), una medida de estímulo no convencional que consiste en la compra de activos de deuda pública por parte de un banco central para aumentar la oferta de dinero. Asimismo, el Banco Central flexibilizó las regulaciones sobre las fintechs, permitiéndoles emitir tarjetas de crédito, recibir fondos directos desde el BNDES (préstamos y transferencias) y permitir que fondos de inversión tomen posesión de estas empresas.

Las políticas empresariales, por su parte, se han dirigido a brindar apoyo financiero (préstamos y subsidios) para los sectores más afectados por la crisis; por ejemplo, se liberaron $\mathrm{R} \$ 5$ billones en créditos para el sector turismo, de los cuales se espera que el $80 \%$ se dirija a pequeños y medianos empresarios. Brasil ha impulsado varias resoluciones encaminadas a la apertura de créditos general; sin embargo, vale resaltar el Programa de Apoio ao Setor Sucroalcooeiro - PASS, anunciado 
como un apoyo específico para el sector de la agroindustria azucarera. "El PASS pondrá a disposición, junto con recursos de bancos comerciales, $\mathrm{R} \$ 3$ billion ( $\mathrm{R} \$ 1,5$ billon del BNDES)" (CEPAL, 2020b). Lo anterior es interesante cuando más allá de reconocer que esta industria emplea cerca de 1 millón de brasileros, se reconoce que "la industria azucarera está cada vez más concentrada en pocas familias, conocidas en Brasil como los barones del azúcar, y en unas cuantas compañías extranjeras, que habitualmente actúan asociadas entre sí" (GRAIN, 2009). De igual forma se establecieron acciones focalizadas al sector de transporte, aviación y minería. De hecho, se negoció un paquete de emergencia para los distribuidores de energía.

Empero, el Índice de Confianza del Empresario Industrial se situó en los 60,3 puntos este mes de marzo, lo que representa 4,4 puntos menos que febrero y la mayor caída desde junio de 2018, según el último informe elaborado por la Confederación Nacional de la Industria (CNI). Lo anterior, a pesar de la estrecha cercanía que el Gobierno de Bolsonaro tiene con los empresarios; incluso, llegó a afirmar que "la libertad es más importante que la vida, al reclamar ante la corte suprema, acompañado por empresarios del sector productivo, la flexibilización de las cuarentenas en estados y ciudades contra la pandemia y advertir sobre un colapso económico y social" (El Clarín, 2020) poniendo de relieve la importancia de priorizar las medidas para mantener a flote al sector empresarial del país.

\section{Consideraciones finales}

La crisis causada por la COVID-19 ha tenido impactos importantes en términos económicos. Lo anterior, sumado a la inestabilidad sociopolítica y las malas perspectivas económicas que ya se tenían antes de que llegara el coronavirus, han profundizado aún más la desigualdad estructural, pues como se mencionó anteriormente, la crisis profundiza las vulnerabilidades y desigualdades. En ese escenario, la efectividad de varios de los instrumentos y acciones que se han implementado se ha visto condicionada por la compleja situación de los países: altas tasas de informalidad, baja penetración bancaria en la economía, así como una elevada población desocupada y los ya conocidos problemas de corrupción. 
Existen varios estudios en donde se señala que el desempeño y desarrollo de los países depende en gran medida de las relaciones y lazos que existen entre el sector público y el sector privado (Schneider and Wolfson, 1999, p. 2). Empero, para que existan estos resultados se requiere de reciprocidad por parte del sector empresarial para poder redistribuir los beneficios. Las relaciones con el sector privado, si bien pueden ser oportunidades, también plantean problemas frente a cómo definir las prioridades en las decisiones políticas. Así, los empresarios, grupos y asociaciones empresariales aparecen como grupos de poder con fuerte incidencia en áreas clave del Estado y las políticas públicas, con alta eficacia en la defensa de sus intereses económicos (Serna and Bottinelli, 2018, p. 10). Esto se ha visto reflejado en varias de las acciones implementadas en los casos de Brasil y Colombia pues, aunque debe resaltarse que se está haciendo un esfuerzo importante para implementar los programas de apoyo al ingreso de las familias vulnerables, también se han visto que los intereses de algunos sectores económicos priman sobre otros. Es decir, "la conformación de estructuras de poder económico y político concentradas, y la debilidad de la sociedad civil terminan configurando un sistema de captura política a pesar de la existencia de instituciones y reglas democráticas" (Durand, 2016, p. 27). Esto, sumado al poder del ejecutivo en un estado de urgencia/emergencia, facilita que se privilegien ciertos intereses. Si bien aún no podemos señalar que exista una clara captura del Estado, la priorización del sector agroindustrial azucarero y de energías en Brasil, o la distribución de las líneas de crédito en Colombia, son muestra de la gran influencia que tienen las élites en estos países para influir en la toma de decisiones y políticas.

Como se mostró en el desarrollo del documento, Brasil y Colombia han desarrollado varios programas y medidas. Empero, al analizar los recursos comprometidos, se evidencia que pese a tener el mayor número de medidas, los recursos públicos puestos a disposición son tímidos comparados con países como Chile, El Salvador y Perú (Pineda, Pessino and Rasteletti, 2020). Además, si bien la posición de los gobiernos de Brasil y Colombia difiere frente a la forma de afrontar la pandemia por la enfermedad de la COVID-19, en ambos casos se ha identificado que las ayudas o han estado dirigidas a los grandes gremios o sistemáticamente 
están dirigidas al beneficio de un grupo minoritario, priorizando el empresariado tradicional y evidenciando que aún en esta coyuntura, las prácticas de corrupción y el aumento de la desigualdad siguen manifestándose.

\section{Bibliografía}

Ávila, R. (2020) La corrupción en Colombia, un mal más grave que el coronavirus, Portafolio. co. Disponible en: https://www.portafolio.co/economia/la-corrupcion-encolombia-un-mal-mas-grave-que-el-coronavirus-545299 (Último acceso: 26 de septiembre de 2020).

Cano, C. G. et al. (2014) 'Inclusión financiera en Colombia', 2014, p. 50.

Carrington, D. (2020) 'Polluter bailouts and lobbying during Covid-19 pandemic', The Guardian, 17 April. Disponible en: http://www.theguardian.com/environment/2020/apr/17/polluter-bailouts-and-lobbying-during-covid-19-pandemic (Último acceso: 13 de julio de 2020).

CEPAL (2019) Persiste la desaceleración generalizada en América Latina y el Caribe en 2019 y se espera un bajo crecimiento para 2020. CEPAL. Disponible en: https:// www.cepal.org/es/comunicados/persiste-la-desaceleracion-generalizadaamerica-latina-caribe-2019-se-espera-un (Último acceso: 7 de julio de 2020).

CEPAL (2020a) América Latina y el Caribe ante la pandemia del COVID-19. Efectos económicos y sociales. Reporte Especial Covid-19 1. Chile: CEPAL.

CEPAL (2020b) COVID-19, Observatorio COVID-2019 América Latina y el Caribe. Comisión Económica para América Latina y el Caribe. Disponible en: https:// www.cepal.org/es/temas/covid-19 (Último acceso: 14 de julio de 2020).

CEPAL (2020c) 'Panorama Fiscal de América Latina y el Caribe 2020. La política fiscal ante la crisis derivada de la pandemia de la enfermedad por coronavirus (COVID-19)'.

Contraloría General de la República (2020) Contraloría alerta sobre concentración de créditos subsidiados para el agro en emergencia por Covid-19 - Boletines de Prensa - 2020 -, Contraloría General de la República. Disponible en: https://www.contraloria.gov.co/contraloria/sala-de-prensa/boletines-deprensa/boletines-de-prensa-2020/-/asset_publisher/9IOzepbPkrRW/content/ contraloria-alerta-sobre-concentracion-de-creditos-subsidiados-para-elagro-en-emergencia-por-covid-19?inheritRedirect=false\&amp; redirect $=$ ht tps $\% 3 \mathrm{~A} \% 2 \mathrm{~F} \% 2 \mathrm{Fwww}$.contraloria.gov.co\%3A443\%2Fcontraloria $\% 2 \mathrm{Fsala}-$ de-prensa\%2Fboletines-de-prensa\%2Fboletines-de-prensa-2020\%3Fp_p_ id\%3D101_INSTANCE_9IOzepbPkrRW\%26p_p_lifecycle\%3D0\%26p_p_ state\%3Dnormal\%26p_p_mode\%3Dview\%26p_p_col_id\%3D_118_ INSTANCE_UixrmFOfHuSj__column-1\%26p_p_col_pos\%3D1\%26p_p_col_ count\%3D2 (Último acceso: 18 de julio de 2020).

Controladoria-Geral da União (2020) CGU monitora aplicação dos recursos federais repassados a estados e municípios, Controladoria-Geral da União. Disponible en: https://www.gov.br/cgu/pt-br/coronavirus/cgu-monitora-aplicacao-dosrecursos-federais-repassados-a-estados-e-municipios (Último acceso: 25 de octubre de 2020).

DANE (2020a) Balanza comercial. Disponible en: https://www.dane.gov.co/index.php/ estadisticas-por-tema/comercio-internacional/balanza-comercial (Último acceso: 21 de octubre de 2020).

DANE (2020b) IndicadoresRelevantes. Disponible en: https://sitios.dane.gov.co/ indicadores-relevantes/ (Último acceso: 21 de octubre de 2020).

Decreto 444 (2020) Decreto 444 de 2020 - EVA - Función Pública. Disponible en: https:// www.funcionpublica.gov.co/eva/gestornormativo/norma.php?i=110678 (Último acceso: 21 de octubre de 2020). 
DeJusticia (2020) 'El Programa Ingreso Solidario estaría excluyendo a mujeres en condiciones críticas de pobreza', Dejusticia, 22 de octubre de. Disponible en: $\quad$ https://www.dejusticia.org/el-programa-ingreso-solidario-estariaexcluyendo-a-mujeres-en-condiciones-criticas-de-pobreza/ (Último acceso: 25 de octubre de 2020).

Deutsche Welle (DW) (2020) 'Brasil se encamina a la peor crisis económica de su historia | DW | 19.05.2020', DW.COM, 22 June. Disponible en: https://www. dw.com/es/brasil-se-encamina-a-la-peor-crisis-econ\%C3\%B3mica-de-suhistoria/a-53500282 (Último acceso: 18 de julio de 2020).

Durand, F. (2016) Cuando el poder extractivo captura El Estado. OXFAM. Perú: OXFAM.

El Clarín (2020) Desde 'gripezinha' a 'la vida continúa': las frases con las que Jair Bolsonaro minimizó el coronavirus - Clarín. Disponible en: https://www. clarin.com/mundo/gripezinha-vida-continua-frases-jair-bolsonaro-minimizocoronavirus_0_aVXRIiYBn.html (Último acceso: 26 de octubre de 2020).

El Tiempo (2020) 'Fiscalía inicia inspección judicial en Finagro', El Tiempo, 13 May. Disponible en: https://www.eltiempo.com/justicia/investigacion/ fiscalia-inspecciona-sede-de-finagro-por-investigacion-por-creditos-porcoronavirus-494958 (Último acceso: 18 de julio de 2020).

Galán, J. (2020) 'DÍAS SIN IVA EN COLOMBIA: ¿CUÁL ES EL IMPACTO ECONÓMICO?', Universidad Sergio Arboleda, 21 May. Disponible en: https://www. usergioarboleda.edu.co/noticias/dias-sin-iva-en-colombia-cual-es-elverdadero-impacto-economico/ (Último acceso: 20 de octubre de 2020).

Gallego, J. A., Prem, M. and Vargas, J. F. (2020) Corruption in the Times of Pandemia. SSRN Scholarly Paper ID 3600572. Rochester, NY: Social Science Research Network. DOI: $10.2139 /$ ssrn.3600572.

GRAIN (2009) Brasil y los nuevos emporios azucareros. Disponible en: https://www. grain.org/es/article/entries/1218-brasil-y-los-nuevos-emporios-azucareros (Último acceso: 18 de julio de 2020).

Hernandez, M. (2020) 'Inestabilidad política y coronavirus: Brasil se encamina hacia su peor contracción económica', France 24, 10 May. Disponible en: https:// www.france24.com/es/20200510-inestabilidad-politica-coronavirus-brasilencamina-peor-contraccion-economica (Último acceso: 18 de julio de 2020).

Klein, N. (2008) La doctrina del shock. El auge del capitalismo del desastre. 1st edn. Argentina: Paidós. Disponible en: https://circulosemiotico.files.wordpress. com/2018/08/klein-naomi-teorc3ada-del-schock.pdf (Último acceso: 13 de julio de 2020$)$.

La República (2020) El Banco de la República puso la tasa más baja de la historia y está por debajo de la inflación. Disponible en: https://www.larepublica.co/economia/ el-banco-de-la-republica-puso-la-tasa-mas-baja-de-la-historia-y-esta-pordebajo-de-la-inflacion-3065708 (Último acceso: 28 de septiembre de 2020).

Mejía, L. F. (2020) 'COVID-19: costos económicos en salud y en medidas de contención para Colombia'. Disponible en: http://www.repository.fedesarrollo.org.co/ handle/11445/3920 (Último acceso: 13 de julio de 2020).

Morales, M. (2020) Así aplicarán los 3 días sin IVA para reactivar la economía, El Tiempo. Disponible en: https://www.eltiempo.com/economia/sectores/iva-colombia-seadelantan-los-tres-dias-sin-impuesto-para-reactivar-economia-496828 (Último acceso: 21 de octubre de 2020).

Observatorio Fiscal de la Pontifica Universidad Javeriana (2020) 'La transparencia en el uso de los recursos para atender la emergencia'. Disponible en: https:// c899837a-b1ec-41b5-9d46-3e957755d77b.filesusr.com/ugd/e33cdb_23b8487 9a27c43f9b641c75cbdd8bd04.pdf (Último acceso: 21 de septiembre de 2020).

Pineda, E., Pessino, C. and Rasteletti, A. (2020) 'Política y gestión fiscal durante la pandemia y la post-pandemia en América Latina y el Caribe', Gestión fiscal, 21 April. Disponible en: https://blogs.iadb.org/gestion-fiscal/es/politica-y-gestionfiscal-durante-la-pandemia-y-la-post-pandemia-en-america-latina-y-el-caribe/ (Último acceso: 26 de octubre de 2020). 
PNUD (2020) Human Development Reports | United Nations Development Programme. Disponible en: http://hdr.undp.org/ (Último acceso: 26 de octubre de 2020).

Portafolio (2020a) Economía colombiana registró preocupante retroceso en agosto, Portafolio.co. Disponible en: https://www.portafolio.co/economia/colombianaregistro-preocupante-retroceso-en-agosto-545804 (Último acceso: 21 de octubre de 2020).

Portafolio (2020b) Los deudores no sienten baja de tasas de interés del Emisor. Disponible en: https://www.portafolio.co/economia/finanzas/los-deudores-no-sientenbaja-de-tasas-de-interes-del-emisor-541609 (Último acceso: 25 de septiembre de 2020).

Presidencia de la República de Colombia (2020a) Con capitalización del Fondo Nacional de Garantías, Gobierno ofrecerá $\$ 70$ billones en créditos para apoyar a distintos sectores de la economía, por coronavirus. Disponible en: https:// id.presidencia.gov.co/Paginas/prensa/2020/Con-capitalizacion-del-FondoNacional-de-Garantias-Gobierno-ofrecera-70-billones-en-creditos-para-apoyara-distintos-200327.aspx (Último acceso: 25 de octubre de 2020).

Presidencia de la República de Colombia (2020b) En primer día sin IVA en Colombia, ventas del comercio superaron los \$5 billones: DIAN, Presidencia de la República. Disponible en: https://id.presidencia.gov.co/Paginas/prensa/2020/ En-primer-dia-sin-IVA-en-Colombia-ventas-del-comercio-superaron-los-5billones-DIAN-200619.aspx (Último acceso: 21 de octubre de 2020).

Presidencia de la República de Colombia (2020c) <Esperamos que primer día sin IVA nos ayude a recuperar la demanda y la economía del país': Presidente Duque, Presidencia de la República. Disponible en: https://id.presidencia.gov. co/Paginas/prensa/2020/Esperamos-que-primer-dia-sin-IVA-nos-ayude-arecuperar-la-demanda-y-la-economia-del-pais-Presidente-Duque-200616.aspx (Último acceso: 20 de octubre de 2020).

Presidencia de la República de Colombia (2020) Gobierno confirma que los tres días sin IVA se realizarán el 19 de junio y el 3 y 19 de julio en todo el país, Presidencia de la República. Disponible en: https://id.presidencia.gov.co/Paginas/ prensa/2020/Gobierno-confirma-que-los-tres-dias-sin-IVA-se-realizaran-el-19de-junio-y-el-3-y-19-de-julio-en-todo-el-pais-200518.aspx (Último acceso: 26 de octubre de 2020).

Presidencia de la República de Colombia (2020d) Presidente Duque afirma que se debe avanzar más en las ayudas a las microempresas del país, Presidencia de la República. Disponible en: https://id.presidencia.gov.co/Paginas/prensa/2020/ Presidente-Duque-afirma-que-se-debe-avanzar-mas-en-las-ayudas-a-lasmicroempresas-del-pais-200417.aspx (Último acceso: 15 de octubre de 2020).

Real Instituto Elcano (2020) El COVID-19 en América Latina: desafíos políticos, retos para los sistemas sanitarios e incertidumbre económica - Elcano. Disponible en: http://www.realinstitutoelcano.org/wps/portal/rielcano_es/contenido?WCM_ GLOBAL_CONTEXT=/elcano/elcano_es/zonas_es/ari27-2020-malamud-nunezcovid-19-en-america-latina-desafios-politicos-retos-sistemas-sanitariose+incertidumbre-economica (Último acceso: 26 de septiembre de 2020).

Revista Semana (2020) 'Democracia con respirador', Revista Semana, 25 April. Disponible en: https://www.semana.com/mundo/articulo/el-coronavirus-infecta-lademocracia-miedo-autoritarismo-y-limites-de-libertad/665966 (Último acceso: 13 de julio de 2020).

Schneider, B. R. and Wolfson, L. (1999) 'Las relaciones entre el estado y las empresas y sus consecuencias para el desarrollo: una revisión de la literatura reciente', Desarrollo Económico, 39(153), p. 45. DOI: 10.2307/3467220.

Serna, M. and Bottinelli, E. (2018) El poder fáctico de las élites empresariales en la política latinoamericana: Buenos Aires: CLACSO - OXFAM.

Souza, A. de (2020) Brasil: doce hospitales temporales son investigados por corrupción, Salud con lupa. Disponible en: https://saludconlupa.com/series/la-segundaola/brasil-doce-hospitales-temporales-son-investigados-por-corrupcion/ (Último acceso: 5 de octubre de 2020). 
Superintendencia Financiera de Colombia (SFC) (2020) Informe sobre el sistema financiero durante la Cuarentena Obligatoria por la vida. Disponible en: https:// www.superfinanciera.gov.co/jsp/10103679 (Último acceso: 25 de septiembre de 2020).

Transparencia Internacional (2020a) '3er reporte»Ciudadanía Activa» Seguimiento al manejo de recursos para la atención a la emergencia del COVID-19: "Estrategia transparencia salva vidas"'. Disponible en: https://transparenciacolombia. org.co/Documentos/Publicaciones/gestion-publica/3-rep-CiudadaniaActiva-21-08-20.pdf (Último acceso: 21 de octubre de 2020).

Transparencia Internacional (2020b) 'CIUDADANÍA ACTIVA CON LOS OJOS PUESTOS EN LOS RECURSOS COVID-19: Segundo reporte de seguimiento a la contratación en el marco de la emergencia del COVID-19'. Disponible en: https:// transparenciacolombia.org.co/Documentos/Publicaciones/gestion-publica/ Segundo-reporte-Ciudadania-Activa.pdf (Último acceso: 21 de octubre de 2020). University of Oxford (2020) Government Response Tracker (OxCGRT), CORONAVIRUS GOVERNMENT RESPONSE TRACKER. Disponible en: https://www.bsg.ox.ac.uk/ sites/default/files/2020-05/BSG-WP-2020-032-v6.0.pdf (Último acceso: 14 de julio de 2020). 